\title{
Adverse effects of laser hair removal
}

\author{
Paloma Tejero $^{1,}$, , Victoria Sunkel ${ }^{2}$, María Victoria Zamorano ${ }^{2}$ \\ ${ }^{1}$ Universidad de Alcalá de Henares, Madrid, Spain \\ ${ }^{2}$ Mediestetic Clinic, Madrid, Spain
}

Email address:

palomatejero@mediestetic.com (P. Tejero), vickysunkel@gmail.com (V. Sunkel), mavi442@hotmail.com (M. V. Zamorano)

\section{To cite this article:}

Paloma Tejero, Victoria Sunkel, María Victoria Zamorano. Adverse Effects of Laser Hair Removal. Journal of Surgery. Special Issue: Breakthroughs in Aesthetic Medicine. Vol. 3, No. 1-1, 2015, pp. 18-20. doi: 10.11648/j.js.s.2015030101.16

\begin{abstract}
Adverse effects observed in 300,000 medical laser hair removal sessions between June 1998 and April 2014 have been indirectly surveyed. The small number of adverse effects and their resolution with no significant sequelae is confirmed. Laser hair removal is a safe and effective method, but it is not risk-free. It is clearly necessary to i) strictly update the patient's medical history at each session, ii) duly train healthcare staff, and iii) follow the applicable safety protocols. Note that data collection was based on the memory of the treating team, which means that significant bias is likely. However, bias will be high for mild adverse effects but very low for serious adverse effects (which have also required follow-up). The conclusions of this work may only be applicable to the moderate and serious adverse effects of laser hair removal, but not to standard, mild adverse effects.
\end{abstract}

Keywords: Adverse Effects, Laser Hair Removal, Paradoxical Growth, Hirsutism

\section{Introduction}

Selective photothermolysis performed with various types of lasers has marked an important step in efforts to eradicate unwanted hair. The introduction of laser technology in hair removal systems has been a significant step forward in destroying the hair follicle, enabling effective hair removal. We have been using Alexandrite lasers in our hair removal treatments since 1998, and in 2003 we added pulsed light systems, as they are highly versatile (especially for facial use).

However, it is well known that both technical and medical issues can arise during laser hair removal treatments. Although it is an effective, convenient and quick form of hair removal, it is not always painless, and "there are some confirmed side effects and other presumed but unconfirmed adverse effects requiring clarification" [1].

In this review, our purpose has been to i) indirectly estimate the frequencies of adverse effects recorded during the 300,000 medical laser hair removal sessions performed by our team at Mediestetic centers between June 1998 and April 2014, ii) determine their cause, and iii) assess their evolution over time, in order to propose measures for prevention and/or treatment of complications.

\section{Material and Methods}

A literature search (PubMed) was carried out using the following search terms: "laser hair removal", "laser epilation", "side effects", "adverse effects", and "complications". Boolean operators were used to make up the search algorithm: (laser hair removal OR laser epilation) AND (side effects OR complications OR adverse effects). Detailed searches were subsequently added with each group of side effects: "laser hair removal", "laser epilation", "hyperpigmentation", "hypopigmentation", "paradoxical hypertrichosis", "burn", "photosensitivity”, "fox fordyce”, "hyperhidrosis", "pain", "leucotrichia”, "infections", "ocular".

A very simple, self-referential questionnaire was given to our medical staff, who indicates and oversees every hair removal treatment, and to our auxiliary staff, who performs every hair removal treatment. The questionnaire was fulfilled by every member of our staff: 5 physicians and 12 assistants. The sample of this study were these 17 questionnaires $(n=17)$. The questionnaire included three simple questions (Fig. 1).

Responses have been analyzed and compared to: a) data published by our group in 2005 [2], and to b) data published from general medical literature.

\footnotetext{
\#1. What adverse effects have you observed during the past 15 years? \#2. Why do you think they occurred? $\#$. What do you think we can do to prevent them?
} 


\section{Results}

Medical and assistant team answers to the questionnaire:

Question \#1 What adverse effects have you observed during the past 15 years?

The most frequent adverse effect of laser hair removal and which is more challenging to manage is burns, which result in changes in pigmentation (usually hypopigmentation), lasting a long time and concerning patients.

Pain during treatment is also an adverse effect described by many patients, sometimes requiring treatment discontinuation, especially in the inguinal and genital areas.

A temporary increase in hairiness can also be a side effect in facial treatments, potentially prompting complaints by patients.

Some rash-like reactions which disappear spontaneously have been described.

Three patients have described increased sweating and bromhydrosis, which disappeared spontaneously.

Two cases of folliculitis barbae after hair removal have been reported.

\section{Question \#2 Why do you think they occurred?}

Most adverse effects of laser hair removal are due to excessive confidence developed after routine performance of these treatments. Sometimes, patients who should not undergo laser hair removal (because they have a tan or because they take certain medication, etc.) end up undergoing treatment anyway because medical staff give in to the pressure exerted by the patient herself or other circumstances.

In the case of increased hairiness, the patient's hormonal status can also be a significant contributing factor.

The cause of sweating disorders is unknown.

Question \#3 What do you think we can do to prevent them?

A good medical history, not only at the start but also associated with a short interview every time the patient will undergo treatment, would help alert us about the patient's condition, as every hair removal session can be different.

\section{Discussion}

It should be taken into account that data collection is based on the memory of the treating team. This means that significant bias is likely. However, bias will be high for mild adverse effects but very low for serious adverse effects (which have also required follow-up). The conclusions of this work may only be applicable to the moderate and serious adverse effects of laser hair removal, but not to mild adverse effects.

The answers by all members of the team have been contrasted, and non-mild adverse effects have been reviewed with each of them.

In 2005, we published the following: "Today, with almost 10 years' experience, we know that it is a safe technique when in good hands, which will continue to expand and reach all segments of the population, but which does meet all of the expectations placed on it: duration, number of sessions, pain, light hairs and white hairs are currently the challenges still to be overcome" [3].
Temporary erythema and post-inflammatory hyperpigmentation are the most frequent adverse effects, just like in most authors reviewed [4,5]. A similar situation is observed in our literature review $[6,7]$.

The occurrence of adverse effects varies greatly based on anatomical location: no significant facial complications have been observed, while the most serious adverse effects occur on limbs, arms and legs.

These results are similar to those described by authors such as Ash [8] and Nanni [9].

We also described 5 patients with increased facial hairiness after hair removal in the first few sessions, which was successfully removed in subsequent sessions by increasing pulse intensity, and stated: "This experience has also been described by other authors [10], some of whom even suggested that laser radiation at low doses may have a stimulating effect. This is the effect currently referred to as paradoxical growth, whose pathogenesis has not been completely established yet [11,12].

In 2014, paradoxical growth on the face and neck in hirsute women is still an issue associated with low dose [13] and hormonal changes.

Based on our experience, the number of adverse effects is getting smaller and smaller with respect to the very high number of patients who undergo laser hair removal treatments.

Between 2005 and 2014, we have observed only one serious case of burns on both legs in a patient with phototype III/IV, who initiated legal action, resulting in a favorable outcome. The patient had had her hair removed in previous sessions resulting in erythema and small burns. For this session, she arrived at the office with her legs fully covered in EMLA.

Regarding the answers in our team:

Most treated patients have phototype II and III, and a few have phototype IV, with no significant differences in treatment acceptance and quality due to phototype.

We have indeed observed, as described by several authors, a highly significant increase in the occurrence of adverse effects in the summer and more so in autumn, on skin areas exposed to the sun, even having used sunscreen and working on skins which had apparently lost their tans and even one month after the last day of sun exposure. This, points to a need to maximize precautions in patients and on areas which have been exposed to the sun, even slightly.

As stated by Dr. Benitez [13], it is advisable to wait at least two to three months after UVA exposure.

It is worth noting, however, that very few unwanted side effects appear on the face, possibly due to the special features of the skin and because patients usually step up sun protection in this area, compared to the occurrence of more severe issues on limbs, arms and legs.

A review of current literature on adverse effects reveals cases of both axillary and inguinal bromhydrosis, and authors agree that it is temporary $[14,15]$.

We have not observed any ocular adverse effects, although it is described in the literature reviewed [16].

The genital area requires special attention as well, since it usually exhibits greater pigmentation and sensitivity to pain 
[17]. Patients should be clearly informed of the greater risk of burns, and performance parameters should be modified, lowering the dose, stretching the area, and applying cold.

In terms of treatment, we have used emollients, topical corticosteroids and polarized light in order to prevent sequelae after the occurrence of burns. Depigmentation therapy was only necessary in a few patients. In all cases, the need for sun protection was stressed.

In cases of paradoxical growth, patients were tested for hormonal pathologies. The result was negative in practically all patients, but a high prevalence of PCOS was found. In some cases, eflornithine was recommended, with no significant improvement. A strict protocol has been established for marking the area to be treated and cooling the surrounding areas.

Laser hair removal is still a safe and effective method, but it is not risk-free, and it requires significant training and regular maintenance of safety protocols.

Burns and their sequelae are the most common adverse effects, requiring not only a medical history and patient inquiry, but also according to some authors [18] the use of preventative corticosteroids after treatment.

\section{References}

[1] Zaragoza Zubira. Depilación Láser. Revista SEME. No 48 Sept. 99; 8-13.

[2] Tejero PG, Martin CM.: "Fotodepilación: Estudio retrospectivo de efectos secundarios, complicaciones y tratamientos en zonas difíciles". Boletín SELMQ 2005 18; 2.

[3] Nanni C., Alster T. "Long Pulser Alexandrite Laser-assisted hair remoud comparisons at 5, 10, 20 millisecond pulse durations"' Comunicación al. American society for laser medicine and surpory Eighteenth annud meeting San Diego, California April 5, 7; 1998.

[4] Boss WK Jr., Usal H., Thompson RC., Fiorillo MA. "A comparison of the long-pulse and short-pulse Alexandrite laser hair removal systems". Ann Plast Surg 1999 April; 42 (4): $381-4$.
[5] Lim SP ${ }^{1}$, Lanigan SW. A review of the adverse effects of laser hair removal. Lasers Med Sci. 2006 Sep; 21(3):121-5.

[6] Gan $\mathrm{SD}^{1}$, Graber EM. Laser hair removal: a review. Dermatol Surg. 2013 Jun;39(6):823-38.

[7] Ash K., Loord J., Newman J., Mc Daniel D., "Hair removal using a long-pulsed Alexandrita Laser". Dermatologic Clinic. Volumen 17. № 2 April 1999; 387-399.

[8] Nanni C., et al. A Laser-assited hair removal: side effects of Qswitched Nd-: YAG, long-pulsed ruby, and alexandrite Leaser. J. Am Acad Dermatol. 1999 Augo; 41 (2 Pt 1): 165:71.

[9] Royo J. "Experiencia en Fotodepilación con el sistema de luz pulsada intensa Epi Light". Light news, vol 3, n 4, Octubre 1997.

[10] Desai $\mathrm{S}^{1}$, Mahmoud BH, Bhatia AC, Hamzavi IH Paradoxical hypertrichosis after laser therapy: a review. Dermatol Surg. 2010 Mar;36(3):291-8. .

[11] Lolis $\mathrm{MS}^{1}$, Marmur ES Paradoxical effects of hair removal systems: a review.J Cosmet Dermatol. 2006 Dec;5(4):274-6.

[12] Benitez Roig V. "Experiencia en Fotodepilación con el sistema de luz pulsada intensa Epi Light". Light news, vol 3, no 4, Octubre 1997.

[13] Vélez González, M. Fotodepilación Áreas de difícil tratamiento Cirugía Plástica Ibero-Latinoamericana - Vol. $36-\mathrm{N}^{\circ} 2$ de 2010

[14] Hélou J ${ }^{1}$, Soutou B, Jamous R, Tomb RAnn Dermatol Venereol. [Novel adverse effects of laser-assisted axillary hair removal].2009 Jun-Jul; 136(6-7):495-500.

[15] Helou $\mathrm{J}^{1}$, Habre M, Soutou B, Maatouk I, Ibrahim T, Tomb R.Reversibility of hyperhidrosis post axillary depilatory laser. Lasers Med Sci 2014 Mar; 29(2):717-21.

[16] Shulman $S^{1}$, Bichler IEye (Lond). Ocular complications of laser-assisted eyebrow epilation.2009 Apr; 23(4):982-3.

[17] Vélez González, M. Fotodepilación Áreas de difícil tratamiento Cirugía Plástica Ibero-Latinoamericana - Vol. 36 - No 2 de 2010

[18] Aldraibi $\mathrm{MS}^{1}$, Touma DJ, Khachemoune A. Hair removal with the 3-msec alexandrite laser in patients with skin types IV-VI: efficacy, safety, and the role of topical corticosteroids in preventing side effects. . J Drugs Dermatol. 2007 Jan;6(1):60-6. 